\title{
LA INTEGRACIÓN DE LOS INMIGRANTES A TRAVÉS DE LA COMUNICACIÓN
}

\author{
THE IMMIGRANT INTEGRATION THROUGH COMMUNICATION
}

\author{
Miguel Clemente \\ Pablo Espinosa \\ Universidad de A Coruña, España/Spain \\ miguel.clemente@udc.es \\ pespinosa@udc.es \\ Mercedes Fernández Antón \\ Universidad Complutense, Madrid, España/Spain \\ mfan@edu.ucm.es
}

Recibido/Received: 30/05/2013

Aceptado/Accepted: 23/09/2013

\section{RESUMEN}

La comunicación es fundamental a la hora de conseguir la integración de los inmigrantes. Se parte de la hipótesis de que a mayor nivel de contacto comunicativo de los inmigrantes con personas y elementos de comunicación de la cultura española, mayor nivel de integración; y de que dicho nivel de integración será mayor en los hijos que en los padres. Para ello se creó una encuesta en la que se investigó hasta qué punto los hijos de tres colectivos de inmigrantes (chinos, marroquíes y rumanos) estaban integrados más a la cultura de origen de sus padres o a la española. Se utilizó una muestra de 900 sujetos, 150 padres y 150 hijos de cada nacionalidad. Los resultados indican cómo la mayor integración se produce en el colectivo rumano y la menor en el chino, y cómo los padres están menos integrados que sus hijos. Se discuten los resultados.

\section{PALABRAS CLAVE}

Inmigración, integración, comunicación, comunicación virtual, segunda generación, relaciones padreshijos.

\section{SUMARIO}

1. Introducción. 2. Aspectos demográficos de la inmigración. 3. Adaptación y aculturación de los inmigrantes y sus hijos en España y en Madrid. 4. Método. 5. Resultados. 6. Conclusiones y discusión. Bibliografía.

\footnotetext{
ABSTRACT

Communication is critical in achieving the integration of immigrants. We start from the assumption that higher level of communicative contact between immigrants and Spanish people communication, higher level of integration, and that this level of integration will be greater in the children than in the parents. To research this fact we created a survey that investigated the extent to which children of three immigrant groups (Chinese, Moroccans and Romanians) were integrated more to the culture of origin of their parents or Spanish. We used a sample of 900 participants, 150 parents and 150 children of every nationality. The results show how greater integration occurs in the Romanian group and lowest in Chinese, and how parents are less integrated than their children. Results are discussed.
} 


\section{KEYWORDS}

Immigration, integration, communication, virtual communication, second-generation, parent-child relationships.

\section{CONTENTS}

1. Introduction. 2. Demographic aspects of immigration. 3. Adaptation and acculturation of immigrants and their children in Spain and Madrid. 4. Method. 5. Results. 6. Second generation. 7. Discrepancies in parent-child. 8. Conclusions and discussion. References.

\section{INTRODUCCIÓN}

Este estudio versa sobre la integración de un tipo perfilado de inmigrantes, en concreto sobre la integración de los hijos de la segunda generación de inmigrantes. Mucho se ha publicado desde el trabajo clásico llevado a cabo por Thomas y Znaniecki, y publicado entre 1918 y 1920, hace casi cien años. Thomas y Znaniecki definieron un concepto pionero para la época, la actitud, y expresan que la actitud es "la contraparte individual del valor social; la actividad, en cualquier forma, es el nexo entre ambas" (1958:22).

\section{ASPECTOS DEMOGRÁFICOS DE LA INMIGRACIÓN}

Este estudio se desarrolló en la Comunidad de Madrid, que posee un porcentaje de población extranjera de un 15,98\% (lo que equivale a 1.047 .174 personas) según se desprende del "Informe de la población extranjera empadronada en la Comunidad de Madrid", elaborado por la Consejería de Asuntos Sociales en 2012 (informe previo). Según los datos recogidos en dicho informe, la evolución de los inmigrantes en la Comunidad de Madrid manifiesta un decrecimiento desde el año 2010, habiéndose dado un crecimiento continuo hasta dicho año. Ha sido el periodo comprendido entre mayo de 2007 y enero de 2008 el de mayor tasa de crecimiento. Temporalmente, tomando como referencia el periodo 2006-2012 (de enero a enero), y considerando que en el primer año analizado el crecimiento fue de un $13.92 \%$, en la actualidad estamos en un nivel de decrecimiento de $5.1 \%$.

La presencia de algunos colectivos en España es un fenómeno poco conocido (Beltrán, 2003) a pesar de su larga historia y del considerable volumen de su población en la actualidad. Así ocurre en el caso de los inmigrantes chinos, ya que si bien una gran mayoría de ellos llevan muchos años en España, se habían convertido en una comunidad "invisible". No ha ocurrido lo mismo con los otros dos colectivos más numerosos en la Comunidad de Madrid, los rumanos y los marroquíes.

Beltrán utiliza un concepto muy importante desde el punto de vista de la integración, el de diáspora. El concepto de diáspora hace referencia a un tipo de migración específico, una de cuyas características es la no asimilación en la sociedad general de acogida. Es decir, los miembros de las comunidades diaspóricas se esfuerzan por no perder ni su lengua materna ni sus vínculos étnicos comunitarios y transnacionales, pues de ello depende su éxito económico y una parte importante de sus actividades laborales. Expresa Beltrán que este concepto se produce más habitualmente en las comunidades asiáticas, si bien se puede detectar en todas, e implica un escollo importante a la hora de conseguir la integración. 
Nadie duda de la alta presencia del colectivo de inmigrantes marroquíes en España, así como en la Comunidad de Madrid; sin duda la cercanía geográfica, así como la valoración de su mano de obra en periodos y actividades muy concretas, han creado en el colectivo imaginario la imagen del mismo. Algo similar ha ocurrido desde hace unos pocos años con el colectivo rumano, que ha desplazado al marroquí de ser el primero en el ranking de inmigrantes, y que debido a la facilidad que poseen para hablar español se pueden integrar de una manera muy adecuada; de hecho, en la Comunidad de Madrid es raro encontrar un negocio de cualquier tipo en el que no haya trabajadores rumanos. Se trata, por lo tanto, de dos tipos de poblaciones inmigrantes que se han hecho indiscutibles en la mente de los madrileños. Sin embargo, a pesar de que los asiáticos están presentes en el territorio español desde hace más de un siglo, su residencia ha pasado prácticamente desapercibida hasta muy recientemente con la excepción de determinadas comunidades como la filipina, ya activa durante la última parte del siglo XIX (Martín, 1998), y otras que desde muy temprano se hicieron notar por las importantes repercusiones económicas de sus actividades aquí desarrolladas, como por ejemplo los indios en las Islas Canarias (Murcia, 1974) o los japoneses en Cataluña y en otros lugares de España (Ramos y Ruiz, 1996; Pareja, 2002). Volviendo al concepto de diáspora, éstas se mantienen a lo largo del tiempo, es decir, sus miembros se adaptan a las sociedades de acogida donde están establecidos sin asimilarse totalmente a ellas, perdiendo su identidad y disolviéndose en la sociedad general (Lie, 1995; Cohen, 1997; Van Hear, 1998; MaMung, 2000).

Desde un punto de vista tradicional, este tipo de diáspora estaba compuesto fundamentalmente por comerciantes (internacionales) y era precisamente en esa ocupación donde más éxito podían tener gracias a la ventaja comparativa de ser biculturales y de formar parte de redes diaspóricas transnacionales (Wong, 1997; Light, 2001). Incluso se han elaborado categorías para describir su funcionamiento: capitalistas parias (pariah capitalists) o minorías intermediarias (middleman minorities), aunque el final de la etapa colonial clásica cuestione el uso actual de ellas (Bonacich, 1973; Chun, 1989; Chirot y Reid, 1997). El capital social internacional que disponen las diásporas proporciona acceso a una confianza asegurada. Así, se relacionan con sus coétnicos repartidos por todo el mundo mediante tratos comerciales asegurándose así que no van a ser engañados (Light 2001; Light y Bachu, 2000).

Respecto a su relación con la sociedad de acogida, a nivel económico se puede afirmar que están insertos en la economía nacional: sus negocios están dados de alta, pagan impuestos $\mathrm{y}$, sobre todo, generan una gran riqueza económica además de dinamizar el comercio internacional español (tanto la importación como la exportación). El hecho de ser biculturales y su resistencia a aculturarse unilateralmente, es decir, a asimilarse en el conjunto de la sociedad general, introduce mayor diversidad cultural a una sociedad que ya de por sí es heterogénea (Beltrán 1997; Beltrán y Sáiz 2002). La mayoría se adapta a la sociedad de acogida y son respetuosos con la ley, lo que no implica que pierdan su identidad.

En definitiva, si los inmigrantes tratan de preservar su lengua y sus valores comunitarios es porque de ello depende su éxito económico y social, y porque su marco de referencia lo constituye la diáspora, a la cual pertenecen, más que el territorio donde se establecen. Es precisamente en sus actividades comerciales y de servicios donde se ofrecen como puentes entre sus países y España, dados sus conocimientos y habilidades biculturales, integrándose con su diversidad en la sociedad de acogida. Todo lo que estamos comentando se agudiza en cuanto que existen más dificultades de adaptación debido a la distancia cultural, cuestión que se cumple en nuestro caso respecto de la comunidad china. La comunidad china está compuesta fundamentalmente por familias que tienden a ser propietarias de sus propios 
negocios (familiares). Las cadenas de migración y los procesos de reunificación familiar facilitan su asentamiento y expansión (Beltrán 1997, 2000, 2002; Beltrán y Sáiz 2002; Nieto 2002).

\section{ADAPTACIÓN Y ACULTURACIÓN DE LOS INMIGRANTES Y SUS HIJOS EN ESPAÑA Y EN MADRID}

La primera generación de inmigrantes de cualquier origen siempre ha sido un colectivo inestable, con gran movilidad y que se inserta en una sociedad, pero todavía no pertenece a ella. En cambio, los hijos de estos inmigrantes nacidos en el país de acogida en su gran mayoría se asientan en la sociedad que recibió a sus progenitores.

La literatura científica al respecto está repleta de estudios sobre la integración, la aculturación, y otras variables de aceptación y rechazo cultural de la población al llegar como inmigrantes a otros países. Sin embargo, apenas existen referencias con respecto a los inmigrantes rumanos o a los marroquíes, ya que ambos expresan una realidad inmigratoria muy cercana a nosotros mismos, pero que no se produce a nivel global. Es por esta razón por lo que la mayoría de los estudios que vamos a mencionar se refieren única y exclusivamente a poblaciones inmigrantes chinas.

Existen importantes diferencias en variables culturales y de personalidad de los inmigrantes y sus hijos con respecto a los individuos del propio país, españoles en este caso. Como muestran Hustinger y Jose (2006) en una revisión de la literatura científica, en dicho estudio, y con respecto a los inmigrantes chinos, tanto niños como adolescentes y adultos de origen chino muestran una mayor contención emocional, inhibición, ansiedad, sensibilidad y cautela comparados con los de origen europeo. Incluso los bebés y niños de corta edad chinos muestran un temperamento más calmado, son menos irritables y lloran y sonríen menos que los europeos. Los rasgos de educación, calma, limpieza, concentración y precisión son inculcados con mayor insistencia por los padres chinos que por los de origen europeo.

Por lo que se refiere a las relaciones paterno-filiales, tras la inmigración, los individuos deben encontrar un equilibrio entre mantener los rasgos de su cultura étnica o adoptar los de la cultura de acogida. Padres e hijos pueden avanzar a través de las distintas situaciones de aculturación a diferente velocidad. Los niveles de aculturación dispares entre padres e hijos pueden aumentar los desafíos normativos en fase de desarrollo de los menores en comparación con las familias con un nivel de aculturación similar entre sus miembros, en particular durante la adolescencia temprana (Costigan y Dokis, 2006). Cuando esta aculturación asimétrica se produce, los hijos suelen asimilar la cultura de acogida más rápido que los padres. Los menores chinos a menudo aprenden el idioma local y absorben la nueva cultura antes que sus padres porque mantienen un contacto regular en la escuela con compañeros y profesores, mientras que los padres a menudo trabajan con otros compañeros chinos y se ven apartados de la cultura dominante (Quin, 2008). Estas diferencias de aculturación pueden producirse tanto en el dominio público (p. ej. uso del lenguaje, consumo de medios), como en el privado (p. ej. adopción de valores individualistas o colectivistas) (Costigan y Dokis, 2006).

Hustinger y Jose (2006) plantean que, en la sociedad china tradicional, el confucionismo ha instaurado la idea de que la personalidad de los niños está principalmente determinada por su entorno. Los padres enseñan a sus hijos a controlar sus emociones y a guardarse sus pensamientos y sentimientos para ellos mismos, además de controlar su comportamiento físico. La cultura china promueve un self o identidad interdependiente, en donde el niño 
aprende a ser receptivo a las necesidades y expectativas del grupo. Los padres chinos enfatizan las obligaciones con la familia y esperan que los adolescentes ayuden, respeten y apoyen a sus familias en mayor medida que los padres occidentales. En este sentido, los conflictos familiares pueden ser especialmente pronunciados en familias de origen chino porque la habitual presión entre los deseos de autonomía del menor adolescente y las normas impuestas por los padres viola las normas culturales chinas de obediencia, respeto, autoridad y armonía familiar (Phinney y Ong, 2002). Otros estudios también han encontrado que los adolescentes de origen chino aspiran a una mayor autonomía de sus padres, aunque a edades posteriores que menores occidentales o de otras minorías (Fuligni, Tseng y Lam, 1999)

La adaptación del estilo educativo de los padres chinos a la cultura dominante, o por el contrario, la adherencia estricta a los valores de importancia de la educación y control paterno existentes en China, repercuten en el nivel de estrés de los adolescentes de origen chino (Quin, 2008). El cambio de perspectiva colectivista a una cultura individualista plantea desafíos considerables para los individuos, lo que puede exacerbar la difícil relación entre padres e hijos durante la adolescencia (Le, Goebert yWallen, 2009). De hecho, una etapa crítica es la adolescencia temprana, en comparación con los periodos de la infancia. En esta etapa, el menor comienza a participar en entornos no elegidos por sus padres y en los que éstos no participan. Esto proporciona a los adolescentes mayores oportunidades de aculturación que cuando eran niños bajo una supervisión más estricta de sus padres. Estas diferencias de aculturación se asocian en los menores de origen chino con una menor competencia social, menor satisfacción con la vida, una mayor ansiedad y una autoestima más baja. Como efecto positivo de las diferencias en la aculturación está la motivación de logro del adolescente para tener una asimilación ascendente en la sociedad de acogida (Costigan y Dokis, 2006).

Un reflejo de las importantes diferencias de aculturación que pueden producirse entre padres e hijos de origen chino está en el hecho de que los menores de origen chino comunican sus actividades personales y sentimientos en menor medida a sus padres que los menores occidentales. El motivo para no comunicar sus actividades es la percepción de que se trata de actividades privadas, que no causan daño a nadie y que sus padres no les escucharían o entenderían (Yau, Tasopoulos-Chan y Smetana, 2009).

El equipo investigador que realiza este informe posee experiencia en la realización de trabajos sobre la integración de los inmigrantes. En su día estudió la integración de los inmigrantes marroquíes en la Comunidad de Madrid, posteriormente la de los inmigrantes chinos, y ahora aborda, en este informe, la integración de la segunda generación. Los trabajos ya realizados se han publicado en parte, y se pueden consultar en Uña, Clemente, Espinosa y Fernández (2008, 2009, 2010, 2011a, 2011b). Por otra parte, uno de los integrantes de este equipo, el profesor Uña, se ha ocupado también del estudio del papel de las variables arquitectónicas y físicas en el encuentro de las culturas, cuestión que se puede consultar en Uña y Bruquetas (2005).

La presente investigación pretende obtener información sobre los padres y los hijos de los inmigrantes rumanos, marroquíes y chinos que residen en la Comunidad de Madrid, es decir, sobre la segunda generación de inmigrantes. Se plantea para ello determinar si existe un choque cultural entre los padres (primera generación de inmigrantes rumanos, marroquíes y chinos en la Comunidad de Madrid) y sus hijos (segunda generación de inmigrantes), determinar si la comunicación (tanto real como virtual) es un factor clave de la integración, y proponer alternativas de integración que permitan superar dicho choque cultural en el caso de 
que exista, y que en líneas generales permitan integrar más adecuadamente a los hijos de los inmigrantes rumanos, marroquíes y chinos en la cultura y tradiciones madrileñas y españolas.

\section{MÉTODO}

Para poder conseguir adecuadamente los objetivos se utilizará una metodología de encuesta, accediendo a una muestra cuya información sea extrapolable a la población a un nivel de error aceptable estadísticamente, y cuyo instrumento primordial será el cuestionario. Se aplicó un cuestionario, en el que la mayoría de las preguntas eran de carácter cerrado, que permitió obtener información relevante referente a las redes de socialización del colectivo y del choque cultural.

La ficha técnica de este estudio es la siguiente:

a) Ámbito: la Comunidad de Madrid

b) Universo: personas de nacionalidad rumana, marroquí o china, o española por nacimiento o por tenerla sus padres, pero que conviven dentro de una unidad familiar en la que los padres son ambos de una de las tres nacionalidades citadas (o de vivir con uno sólo de ellos éste debe ser de una de dichas tres nacionalidades) y que no provienen de un tercer país, residentes en la actualidad en la Comunidad de Madrid, de entre 10 y 25 años, con conocimiento del idioma español, y un tiempo mínimo de residencia de 1 año en España o en la Comunidad de Madrid.

c) Tamaño y distribución de la muestra: se administraron los cuestionarios a un total de 900 personas; 150 padres rumanos, 150 hijos rumanos, 150 padres marroquíes, 150 hijos marroquíes, 150 padres chinos, y 150 hijos chinos; con entrevistas realizadas mediante contacto en calle por encuestadores cualificados (para el acceso a la muestra de rumanos los encuestadores eran de dicha nacionalidad, en el caso marroquí también era marroquí el entrevistador, y en el caso de la muestra de sujetos chinos el entrevistador era chino).

d) Error de muestreo: se siguieron los parámetros del muestreo aleatorio simple, para un nivel de confianza bilateral de $95,5 \%$, es decir de 2 sigmas, bajo la hipótesis más desfavorable (es decir, $\mathrm{p}=\mathrm{q}=0,50$ ). Bajo estas condiciones, el margen de error para la totalidad de la muestra es de $\pm 2,5 \%$ (según la formulación y las tablas de Arkin y Colton).

e) Método de recogida de información: entrevista personal realizada en la calle, en lugares próximos a los sitios de reunión de los hijos de los inmigrantes en la Comunidad de Madrid. Los lugares escogidos fueron: inmigrantes rumanos, en la calle y en cafeterías; inmigrantes marroquíes en la calle y en tiendas de productos marroquíes; y los inmigrantes chinos en los locales de ocio de tipo electrónico (locales de "maquinitas"). Todos los sitios de contacto se establecieron en la zona Centro de la capital.

f) Tratamiento de la información: mediante el programa informático PASW - S.P.S.S 18.0. (Statistical Package for Social Sciences). Codificación, verificación, depuración y tratamiento de los resultados.

g) Fecha del trabajo de campo: desde octubre de 2010 hasta octubre de 2011.

\section{RESULTADOS}

\subsection{Segunda generación}

Varias preguntas nos permiten verificar el nivel de integración de los hijos de los inmigrantes residentes en la Comunidad de Madrid. En la primera se puede observar la importancia que se otorga a la conservación de costumbres y tradiciones (Tabla 1). 
TABLA 1: Mantenimiento de costumbres y tradiciones de 1(nada) a 5 (muchísimo)

\begin{tabular}{|l|c|c|c|c|c|c|}
\hline \multicolumn{1}{|c|}{ Origen } & \multicolumn{2}{|c|}{ Rumano } & \multicolumn{2}{c|}{ Marroquí } & \multicolumn{2}{c|}{ Chino } \\
\hline $\begin{array}{l}\text { ¿Crees que es importante } \\
\text { mantener las costumbres y } \\
\text { tradiciones de tu país? }\end{array}$ & 3,58 & 1,235 & 3,50 & 1,403 & 3,28 & 1,239 \\
\hline $\begin{array}{l}\text { ¿Crees que es importante } \\
\text { mantener las costumbres y } \\
\text { tradiciones de España? }\end{array}$ & 3,54 & 1,057 & 3,48 & 1,110 & 3,03 &, 956 \\
\hline
\end{tabular}

Como se puede observar, todos los sujetos consideran importante el mantenimiento de las costumbres y tradiciones de su país y las españolas en una medida más o menos similar. Las diferencias son mínimas, salvo una opinión ligeramente distinta en el caso de los chinos, que "ligeramente" consideran mejor mantener sus propias costumbres que las españolas. Además, tampoco se siente ninguno de los colectivos especialmente inclinado a la conservación de ninguna de las tradiciones ni costumbres.

TABLA 2: Nivel de vinculación a la cultura propia y española de 1 (Nada) a 5 (Muchísimo): relaciones de amistad (grupo de pares)

\begin{tabular}{|l|c|c|c|c|c|c|}
\hline & \multicolumn{2}{|c|}{ Rumano } & \multicolumn{2}{c|}{ Marroquí } & \multicolumn{2}{c|}{ Chino } \\
\hline & Media & Desv. típ. & Media & Desv. típ & Media & Desv. típ. \\
\hline $\begin{array}{l}\text { ¿Crees importante buscar y mantener } \\
\text { relaciones con otros de tu país? }\end{array}$ & 3,64 & 1,037 & 3,61 & 1,082 & 3,31 & 1,099 \\
\hline $\begin{array}{l}\text { ¿Crees importante buscar y mantener } \\
\text { relaciones con españoles? }\end{array}$ & 3,67 & 1,162 & 3,60 & 1,229 & 3,29 & 1,017 \\
\hline $\begin{array}{l}\text { ¿Cuál es tu grado de contacto con } \\
\text { otros de tu país que viven aquí? }\end{array}$ & 3,59 & 1,081 & 3,55 & 1,108 & 3,33 & 1,171 \\
\hline $\begin{array}{l}\text { ¿Cuál es tu grado de contacto con } \\
\text { otros de tu país que no viven aquí? }\end{array}$ & 3,31 & 1,148 & 3,23 & 1,340 & 3,27 & 1,180 \\
\hline $\begin{array}{l}\text { ¿Cuál es tu grado de contacto con } \\
\text { personas españolas? }\end{array}$ & 3,66 & 1,228 & 3,60 & 1,329 & 3,05 & 1,078 \\
\hline $\begin{array}{l}\text { ¿Te sientes apoyado en tus problemas } \\
\text { por otros de tu país que viven aquí? }\end{array}$ & 3,31 & 1,274 & 3,30 & 1,327 & 3,22 & 1,148 \\
\hline $\begin{array}{l}\text { iTe sientes apoyado en tus problemas } \\
\text { por los españoles? }\end{array}$ & 3,37 & 1,167 & 3,26 & 1,331 & 2,92 & 1,057 \\
\hline
\end{tabular}

La Tabla 2 muestra cómo los tres colectivos consideran igual de importante mantener relaciones de amistad tanto con las personas de sus países como con los madrileños y españolas. Lo mismo se manifiesta a la hora de hablar del grado de contacto con personas de su país y/o madrileños o españoles; las cifras son muy similares. Resulta muy curioso observar además cómo, debido sin duda a la influencia de las nuevas tecnologías, se sienten en contacto de manera similar tanto con personas de su país que no viven con ellos o están cercanos a ellos, como con los que sí están dentro de su entorno espacial cercano. Por último, respecto a si se sienten más apoyados, caso de tener problemas, por los españoles o madrileños por los de sus países de origen, expresan valores de nuevo similares. Por lo tanto, es preciso destacar cómo en lo referente a las relaciones sociales y a sus grupos de pares, la integración es similar tanto con personas de sus países de origen como con los madrileños. 
La Tabla 3 se centra en la integración mediante la utilización de los medios de comunicación, en concreto de la televisión. Los resultados indican cómo todos los colectivos visionan la televisión de sus países, sin duda a través de Internet. Pero además, tanto los colectivos rumanos como marroquíes visionan más televisión española que de sus países, ocurriendo lo contrario en el colectivo chino. Es verdad que las diferencias son pequeñas, pero el colectivo chino se comporta de una manera diferente; prefiere visionar su televisión y le gusta más, al contrario que los otros dos.

TABLA 3: Nivel de vinculación a la cultura propia y española de 1 (Nada) a 5 (Muchísimo): medios de comunicación (TV)

\begin{tabular}{|l|c|c|c|c|c|c|}
\hline \multicolumn{1}{|c|}{ Origen } & \multicolumn{2}{c|}{ Rumano } & \multicolumn{2}{c|}{ Marroquí } & \multicolumn{2}{c|}{ Chino } \\
\hline & Media & Desv. típ. & Media & Desv. típ & Media & Desv. típ. \\
\hline $\begin{array}{l}\text { iVes programas de } \\
\text { televisión de tu país? }\end{array}$ & 3,02 & 1,357 & 2,95 & 1,501 & 3,52 & 1,244 \\
\hline $\begin{array}{l}\text { iTe gusta ver programas } \\
\text { de televisión de tu país? }\end{array}$ & 3,28 & 1,257 & 3,10 & 1,437 & 3,42 & 1,210 \\
\hline $\begin{array}{l}\text { iVes programas de } \\
\text { televisión españoles? }\end{array}$ & 3,58 & 1,220 & 3,60 & 1,178 & 3,18 & 1,131 \\
\hline $\begin{array}{l}\text { iTe gusta ver programas } \\
\text { de televisión españoles? }\end{array}$ & 3,61 & 1,137 & 3,59 & 1,232 & 3,26 & 1,233 \\
\hline
\end{tabular}

Se investigó también si la radio funciona como una fuente de integración, repitiendo las preguntas anteriores pero con respecto a este medio. Los resultados se pueden consultar en la Tabla 4.

TABLA 4: Nivel de vinculación a la cultura propia y española de 1 (Nada) a 5 (Muchísimo): radio

\begin{tabular}{|l|c|c|c|c|c|c|}
\hline \multicolumn{1}{|c|}{ Origen } & \multicolumn{2}{|c|}{ Rumano } & \multicolumn{2}{c|}{ Marroquí } & \multicolumn{2}{c|}{ Chino } \\
\hline & Media & Desv. típ. & Media & Desv. típ & Media & Desv. típ. \\
\hline $\begin{array}{l}\text { ¿Escuchas programas } \\
\text { de radio de tu país? }\end{array}$ & 2,89 & 1,297 & 2,72 & 1,423 & 3,11 & 1,377 \\
\hline $\begin{array}{l}\text { ¿Te gusta escuchar la } \\
\text { radio de tu país? }\end{array}$ & 3,04 & 1,235 & 3,17 & 1,306 & 3,10 & 1,257 \\
\hline $\begin{array}{l}\text { ¿Escuchas programas } \\
\text { de radio españoles? }\end{array}$ & 3,31 & 1,253 & 3,70 & 1,193 & 2,89 & 1,277 \\
\hline $\begin{array}{l}\text { ¿Te gusta escuchar la } \\
\text { radio española? }\end{array}$ & 3,27 & 1,271 & 3,41 & 1,261 & 2,71 & 1,254 \\
\hline
\end{tabular}

Los resultados de la tabla anterior ponen de manifiesto cómo lo especificado para televisión se manifiesta de manera idéntica en el caso de la radio. Los tres colectivos escuchan radio de su país, pero el colectivo de hijos chinos de manera ligeramente superior a la audición de radios españolas. En los otros colectivos escuchan más la radio española que la de sus países, y les gusta más la española. En el colectivo chino ocurre justamente lo contrario. Estamos, por lo tanto, ante un freno a su integración.

Por último, se preguntó lo mismo pero con respecto a Internet, figurando los resultados en la Tabla 5. Los datos muestran cómo una vez más se produce el mismo resultado. Los hijos de los inmigrantes rumanos y marroquíes utilizan más (y en este caso de manera más marcada) contenidos de Internet españoles que de su país de origen. 
TABLA 5: Nivel de vinculación a la cultura propia y española de 1 (Nada) a 5 (Muchísimo): Internet

\begin{tabular}{|l|c|c|c|c|c|c|}
\hline \multicolumn{1}{|c|}{ Origen } & \multicolumn{2}{|c|}{ Rumano } & \multicolumn{2}{c|}{ Marroquí } & \multicolumn{2}{c|}{ Chino } \\
\hline & Media & Desv. típ. & Media & Desv. típ & Media & Desv. típ. \\
\hline $\begin{array}{l}\text { ¿Utilizas contenidos de tu } \\
\text { país en Internet? }\end{array}$ & 3,32 & 1,283 & 2,96 & 1,369 & 3,53 & 1,259 \\
\hline $\begin{array}{l}\text { ¿Te gusta utilizar contenidos } \\
\text { de tu país en Internet? }\end{array}$ & 3,28 & 1,266 & 2,95 & 1,313 & 3,44 & 1,181 \\
\hline $\begin{array}{l}\text { ¿Utilizas contenidos } \\
\text { españoles en Internet? }\end{array}$ & 3,56 & 1,299 & 3,34 & 1,291 & 3,16 & 1,191 \\
\hline $\begin{array}{l}\text { ¿Te gusta utilizar contenidos } \\
\text { españoles en Internet? }\end{array}$ & 3,48 & 1,285 & 3,59 & 1,270 & 2,96 & 1,260 \\
\hline
\end{tabular}

Pero ocurre lo contrario en el caso de los hijos de los inmigrantes chinos; éstos utilizan ligeramente más los contenidos de su propio país de origen.

\subsection{Discrepancias padres-hijos}

A continuación se examinaron las discrepancias entre padres e hijos en lo que se refiere a mantenimiento de la cultura de su país de origen e inmersión en la cultura española. Los contrastes se realizaron a través de pruebas $t$ para muestras relacionadas.

Como se puede verificar en la Tabla 6, las comparaciones entre padres e hijos rumanos muestran cómo los hijos utilizan mucho más Internet que sus padres, les gusta más utilizar los contenidos de Internet de su país, también utilizan más contenidos de Internet en español, y les gustan más los contenidos de Internet en español que a sus padres.

TABLA 6: Discrepancias entre padres e hijos rumanos

\begin{tabular}{|l|l|c|c|c|c|c|}
\hline \multicolumn{7}{|c|}{ Prueba de muestras relacionadas } \\
\hline Origen & $\mathrm{t}$ & $\mathrm{gl}$ & $p$ & Media hijos & Media padres \\
\hline rumano & $\begin{array}{l}\text { ¿Utilizas contenidos de tu } \\
\text { país en Internet? }\end{array}$ & 3,127 & 148 &, 002 & 3,31 & 2,91 \\
\hline & $\begin{array}{l}\text { ¿Te gusta utilizar contenidos } \\
\text { de Internet de tu país? }\end{array}$ & 2,325 & 147 &, 021 & 3,28 & 2,99 \\
\hline & $\begin{array}{l}\text { ¿Utilizas contenidos } \\
\text { españoles en Internet? }\end{array}$ & 4,466 & 148 &, 000 & 3,54 & 2,97 \\
\hline & $\begin{array}{l}\text { ¿Te gusta utilizar contenidos } \\
\text { españoles en Internet? }\end{array}$ & 3,258 & 147 &, 001 & 3,47 & 3,01 \\
\hline
\end{tabular}

Por lo que se refiere a muestra de padres y de hijos marroquíes (Tabla 7), las variables significativas hacen referencia a que los padres tienen mayor grado de contacto con los compatriotas de fuera de España que sus hijos, a que de nuevo los padres se sienten más apoyados por los españoles si tienen problemas, también los padres ven más programas de televisión de su país de origen que sus hijos, y les gusta más verlos. Por otra parte, los hijos escuchan más programas de radio españoles que sus padres, leen más prensa tanto de su país de origen como española que sus padres, y utilizan más Internet que sus padres, tanto con respecto a contenidos de su país de origen como españoles (lo cual puede expresar un problema generacional de acceso a las nuevas tecnologías). Por lo tanto, en general se percibe una culturación mayor de los hijos hacia la cultura española, y consecuentemente una aculturación mayor de los descendientes hacia la cultura de origen. Esta es la información que se puede observar en la tabla siguiente. 
TABLA 7: Discrepancias entre padres e hijos marroquíes

\begin{tabular}{|l|l|c|c|c|c|c|}
\hline \multicolumn{6}{|c|}{ Prueba de muestras relacionadas } \\
\hline Origen & $\mathrm{t}$ & $\mathrm{gl}$ & $p$ & Media hijos & $\begin{array}{c}\text { Media } \\
\text { padres }\end{array}$ \\
\hline marroquí & $\begin{array}{l}\text { Grado de contacto con } \\
\text { compatriotas de fuera de España }\end{array}$ & $-2,404$ & 144 &, 017 & 3,24 & 3,58 \\
\hline & $\begin{array}{l}\text { ¿Te sientes apoyado en tus } \\
\text { problemas por los españoles? }\end{array}$ & $-2,002$ & 142 &, 047 & 3,23 & 3.48 \\
\hline & $\begin{array}{l}\text { ¿Ves programas de televisión de } \\
\text { tu país? }\end{array}$ & $-2,005$ & 143 &, 047 & 2,96 & 3,20 \\
\hline & $\begin{array}{l}\text { ¿Te gusta ver programas de } \\
\text { televisión de tu país? }\end{array}$ & $-2,316$ & 142 &, 022 & 3,07 & 3,36 \\
\hline & $\begin{array}{l}\text { ¿Escuchas programas de radio } \\
\text { españoles? }\end{array}$ & 4,169 & 141 &, 000 & 3,70 & 3,13 \\
\hline & ¿Lees prensa de tu país? & 2,280 & 143 &, 024 & 2,98 & 2,63 \\
\hline & ¿Lees prensa española? & 4,035 & 141 &, 000 & 3,33 & 2,74 \\
\hline & ¿Te gusta leer prensa española? & 2,016 & 138 &, 046 & 3,35 & 3,01 \\
\hline & $\begin{array}{l}\text { ¿Te gusta utilizar contenidos de } \\
\text { tu país en Internet? }\end{array}$ & 2,653 & 144 &, 009 & 2,95 & 2,67 \\
\hline & $\begin{array}{l}\text { ¿Utilizas contenidos españoles en } \\
\text { Internet? }\end{array}$ & 4,387 & 145 &, 000 & 3,36 & 2,75 \\
\hline $\begin{array}{l}\text { ¿Te gusta utilizar contenidos } \\
\text { españoles en Internet? }\end{array}$ & 4,020 & 147 &, 000 & 3,60 & 2,99 \\
\hline
\end{tabular}

Por último, analizamos los resultados de la muestra de sujetos de origen chino. Los datos permiten afirmar (Tabla 8) que los hijos visionan más programas de televisión españoles que sus padres, y escuchan más programas de radio españoles que sus padres. Asimismo, también los hijos utilizan más contenidos españoles de Internet que sus padres, y les gustan más los contenidos de Internet españoles que a sus padres. Por lo tanto, se manifiesta de manera clara una mayor integración en la cultura madrileña y española de los hijos chinos que de sus padres.

TABLA 8: Discrepancias entre padres e hijos chinos

\begin{tabular}{|c|l|c|c|c|c|c|}
\hline \multicolumn{7}{|c|}{ Prueba de muestras relacionadas } \\
\hline Origen & $\mathrm{t}$ & $\mathrm{gl}$ & $p$ & Media hijos & Media padres \\
\hline chino & ¿Ves programas de televisión españoles? & 3,932 & 128 &, 000 & 3,17 & 2,72 \\
\hline & ¿Escuchas programas de radio españoles? & 3,300 & 128 &, 001 & 2,88 & 2,46 \\
\hline & ¿Utilizas contenidos españoles en Internet? & 3,430 & 126 &, 001 & 3,18 & 2,70 \\
\hline & $\begin{array}{l}\text { ¿Te gusta utilizar contenidos españoles en } \\
\text { Internet? }\end{array}$ & 1,999 & 128 &, 048 & 2,95 & 2,67 \\
\hline
\end{tabular}

\section{CONCLUSIONES Y DISCUSIÓN}

Todos los sujetos consideran importante el mantenimiento de las costumbres y tradiciones de su país y las españolas en una medida más o menos similar. Las diferencias son mínimas, salvo una opinión ligeramente distinta en el caso de los chinos, que "ligeramente" consideran mejor mantener sus propias costumbres que las españolas. Además, tampoco se siente especialmente inclinado ninguno de los colectivos a la conservación de ninguna de las tradiciones ni costumbres. 
Los tres colectivos consideran igual de importante mantener relaciones de amistad tanto con las personas de sus países como con los madrileños y españoles. Lo mismo se manifiesta a la hora de hablar del grado de contacto con personas de su país y/o madrileños o españoles; las cifras son muy similares.

Todos los colectivos visionan la televisión de sus países. Además, tanto los colectivos rumanos como marroquíes visionan más televisión española que de sus países, ocurriendo lo contrario en el colectivo chino. El colectivo chino prefiere visionar su televisión y le gusta más, al contrario que los otros dos.

Los tres colectivos escuchan radio de su país, pero el colectivo de hijos chinos de manera ligeramente superior a la audición de radios españolas. En los otros colectivos escuchan más la radio española que la de sus países, y les gusta más la española. En el colectivo chino ocurre justamente lo contrario.

Los hijos de los inmigrantes rumanos y marroquíes utilizan más (y en este caso de manera más marcada) contenidos de Internet españoles que de su país de origen. Ocurre lo contrario en el caso de los hijos de los inmigrantes chinos, que utilizan ligeramente más los contenidos de su propio país de origen.

Esta investigación parte de la idea general de que existe un conflicto generacional entre padres e hijos, prácticamente inherente a la realidad de la convivencia entre generaciones, y derivada de ella, de la idea de que este hecho se verá agravado en el caso de las poblaciones inmigrantes, ya que es previsible que la segunda generación se separe más de las tradiciones culturales de origen, y se integre más en la cultura madrileña y española, por lo que se producirá en mayor medida un conflicto generacional. Es más, se preveía que cuanto mayor distancia cultural haya, mayor será el conflicto intergeneracional, de forma que los hijos de los inmigrantes chinos serán los que supondrán un mayor conflicto familiar, seguidos por los hijos de los inmigrantes marroquíes, y por último de los rumanos, se supone que culturalmente más cercano a nosotros. Añadamos dentro de esta reflexión que dicha clasificación de la distancia entre culturas se apoya en la literatura científica sobre el tema, pero es una clasificación exclusivamente nuestra.

Sin embargo, los datos de este trabajo no avalan lo expresado, sino que ponen de manifiesto cómo existe un nivel de conflicto bajo entre los padres y los hijos, si bien sistemáticamente se verifica que los hijos están más integrados que sus padres. Si bien no tenemos una muestra de comparación de padres e hijos residentes y provenientes familiarmente de la Comunidad de Madrid, por lo que la afirmación que vamos a efectuar no es verificable en su totalidad, consideramos que el conflicto intergeneracional es mayor entre padres e hijos madrileños que el existente en los colectivos analizados. Cabría por lo tanto preguntarse por la razón de que el conflicto sea menor, y creemos que se puede encontrar la respuesta en las prácticas de crianza mucho más restrictivas con sus hijos de los tres colectivos analizados, el que al residir en otro país priman más en los hijos las tradiciones paternas que la influencia de la sociedad en la que están inmersos. Por lo tanto, la segunda generación no es un colectivo más cercano a las tradiciones y cultura madrileñas y españolas, sino a la cultura de sus padres. Y este hecho no se produce por un intento de defender su cultura, sino exclusivamente por haber recibido una socialización al respecto.

Se trata de tres colectivos que valoran la cultura y las tradiciones madrileñas y españolas, con las peculiaridades y límites ya comentados. Que prefieren su cultura ligeramente más que la del lugar de su residencia, pero que avanzan hacia un biculturalismo. Por otra parte, las tres culturas son mucho más colectivistas que la 
madrileña, lo que implica una visión de la realidad bastante diferente a la actual dentro de la Comunidad de Madrid, mucho más individualista. Quizá por esa cuestión tienden más a reunirse dentro de sus propios grupos, enlenteciendo la integración.

Puesto que nuestro objetivo a conseguir es evitar el conflicto entre padres e hijos, y promover la integración de ambos en la sociedad madrileña, se recomienda trabajar en crear un sistema que permita incrementar la red social de los inmigrantes, con personas que no sean de su familia ni sólo de su país, especialmente en el caso del colectivo chino.

\section{BIBLIOGRAFÍA}

BELTRÁN, J. (1997), “Immigrés chinois en Espagne ou citoyens européens?", en Revue Européenne des Migrations Internationales, vol. 13, nº 2, pp. 63-79.

BELTRÁN, J. (2000), "La empresa familiar. Trabajo, redes sociales y familia en el colectivo chino", en Ofrim/Suplementos, vol 6, pp. 129-153.

BELTRÁN, J. (2002), "La diáspora china y la globalización. Allá donde llegan las olas...", en Vanguardia Dossier, vol 2, pp. 82-84.

BELTRÁN, J. (2003), "Diáspora y Comunidades Asiáticas en España", en Scripta Nova: revista electrónica de Geografía y Ciencias Sociales, vol VII, nº 134. en: http://www.ub.edu/geocrit/sn/sn-134. $\mathrm{htm}$, visitado 11/08/2013.

BELTRÁN, J. y SÁIZ, A. (2002), “Comunidades asiáticas en España”, en Documentos CIDOB. Relaciones España-Asia, vol 3, en: http://www.cidob.org/Castellano/Publicaciones/documentos\% 20cidob/pdf/beltran.pdf, visitado 11/08/2013.

BONACICH, E. (1973), “A theory of middleman minorities", en American Sociological Review, vol $38, n^{\circ} 5$, pp. 583-594.

CHIROT, D. y REID, A. (eds.) (1997), Essential outsiders. Chinese and Jews in the modern transformation of Southeast Asia and Central Europe, Seattle, University of Washington Press.

CHUN, A. (1989), "Pariah capitalism and the overseas Chinese of Southeast Asia. Problems in the definition of the problem", en Ethnic and Racial Studies, vol 12, n 2, pp. 233-256.

COHEN, R. (1997), Global Diasporas, Seattle, University of Washington Press.

COSTIGAN, C. L. y DOKIS, D. P. (2006),"Relations between parent-child acculturation differences and adjustment within immigrant chinese families", en Child Development, vol 77, $\mathrm{n}^{\circ}$ 5, pp. 1252-1267. FULIGNI, A.; TSENG, V. y LAM, M. (1999), "Attitudes towards family obligations among American adolescents from Asian, Latin American, and European backgrounds", en Child Development, vol 70, pp.1030-1044

HUSTINGER, C. S. y JOSE, P. E. (2006), “A longitudinal investigation of personality and social adjustment among Chinese American and European American adolescents", en Child Development, vol $77, \mathrm{n}^{\circ} 5$, pp. 1309-1324.

LE, T.N., GOEBERT, D. y WALLEN, J. (2009), "Acculturation factors and substance use among Asian American youth", en Journal of Primary Prevention, vol 30, pp. 453-473.

LIE, J. (1995), "From international migration to transnational diaspora", en Contemporary Sociology, vol 4, pp. 303-306.

LIGTH, I. (2001), “Globalization, transnationalism and trade", en Asian and Pacific Migration Journal, vol 10, n' 1 , pp. 53-79.

LIGHT, I. y BHACHU, P. (eds.) (2000), Immigration and entrepreneurship, New Brunswick, Transaction.

MA-MUNG, E. (2000), La diaspora chinoise. Géographie d'une migration, Paris, Ophrys.

MARTíN, E. (1998), "Filipinos en España en los siglos XIX y XX (1868-1936)", en Cuadernos de Historia. Instituto Cervantes de Manila, vol 2-3, pp. 169-182.

MURCIA, E. (1974), "Los comerciantes hindúes en Santa Cruz de Tenerife”, en Estudios geográficos, $n^{\circ} 136$, pp. 405-442. 
NIETO, G. (2002), "Inmigración china en España: Análisis comparado con otros países europeos”, en Fanjul, E. y Molero, J. (coords.), Asia, una nueva frontera para España, Madrid, Editorial Complutense, pp. 67-85.

PAREJA, P. (2002), "Relaciones político-institucionales entre Cataluña y Asia-Pacífico". Documentos CIDOB. Relaciones España-Asia, 1, 1, en http://www.cidob.org/Castellano/Publicaciones/documentos \%20cidob/pdf/pareja.pdf, visitado 11/08/2013.

PHINNEY, J. S. (1992), “The multigroup ethnic identity measure”, en Journal of Adolescent Research, vol 7, pp. 156-176.

PHINNEY, J. S., y ONG, A. D. (2002), "Adolescent-parent disagreements and life satisfaction in families from Vietnamese and European-American backgrounds", en International Journal of Behavioral Development, vol 26, pp. 556-561.

QIN, D. B. (2008), "Doing well vs. feeling well: understanding family dynamics and the psychological adjustment of Chinese immigrant adolescents", en Journal of Youth and Adolescence, vol 37, pp. 22-35. RAMOS, L. y RUIZ, J. M. (eds.) (1996), Economía y empresa japonesa. Su presencia en España, Valladolid, Secretariado de Publicaciones e Intercambio Científico, Universidad de Valladolid.

THOMAS, W.I. y ZNANIECKI, F.W. (1918-1920), The Polish peasant in Europe and America. Monograph of an immigrant group.

-- (1918), Volume 1: Primary-group organization, Chicago, University of Chicago Press.

-- (1918), Volume 2: Primary-group organization, Chicago, University of Chicago Press.

-- (1919), Volume 3: Life record of an immigrant, Boston, Badger.

-- (1920), Volume 4: Disorganization and reorganization in Poland, Boston, Badger.

-- (1920), Volume 5: Organization and disorganization in America. Boston, Badger.

UÑA, O. y BRUQUETAS, C. (coords.) (2005), El Urbanismo ante el encuentro de las culturas, Madrid, Comunidad de Madrid.

UÑA, O.; CLEMENTE, M.; ESPINOSA, P. y FERNÁNDEZ, M. (2008), "Los valores de los inmigrantes marroquíes en la Comunidad de Madrid", en Sociedad y Utopía, no 32, pp. 97-111.

UÑA, O.; CLEMENTE, M.; ESPINOSA, P. y FERNÁNDEZ, M. (2009), "El tiempo de residencia como factor de integración de los inmigrantes marroquíes en la Comunidad de Madrid”, en Sociedad y Utopía, $\mathrm{n}^{\circ} 33$, pp. 91-104.

UÑA, O.; CLEMENTE, M.; ESPINOSA, P. y FERNÁNDEZ, M. (2010), "Caracterización psicosocial de los hijos de los inmigrantes chinos en la Comunidad de Madrid”, en Sociedad y Utopía, $\mathrm{n}^{\mathrm{o}}$ 36, pp. 67-85.

UÑA, O.; CLEMENTE, M.; ESPINOSA, P. y FERNÁNDEZ, M. (2011a), "Choque cultural y confianza entre padres e hijos inmigrantes chinos", en Sociedad y Utopía, n 37, pp. 81-94.

UÑA, O.; CLEMENTE, M.; ESPINOSA, P. y FERNÁNDEZ, M. (2011b), "La orientación hacia la cultura china y/o española en los hijos de los inmigrantes chinos", en Sociedad y Utopía, $\mathrm{n}^{\mathrm{o}} 38$, pp. 4562.

VAN HEAR, N. (1998), New diasporas. The mass exodus, dispersal and regrouping of migrants communities, Londres, UCL Press.

WONG, L (1997), "Globalization and transnational migration”, en International Sociology, vol 12, pp. 329-351.

YAU, J. P., TASOPOULOS-CHAN, M. y SMETANA, J. G. (2009), "Disclosure to parents about everyday activities among American adolescents from Mexican, Chinese and European backgrounds", en Child Development, vol 80, $\mathrm{n}^{\circ}$ 5, pp. 1481-1498. 


\section{Breve currículo:}

\section{Miguel Clemente}

Catedrático de Psicología Social en la Universidad de A Coruña, en la que es el Director de la Unidad de Investigación en Psicología Jurídica y Protección del Menor. Sus temas de investigación se centran en el estudio de los problemas sociales, especialmente en la integración de los colectivos inmigrantes y en la imitación de la violencia televisiva por parte de los menores.

\section{Pablo Espinosa}

Doctor en Psicología por la Universidad de A Coruña. Ha desarrollado su labor profesional en la universidad de Kent, Reino Unido, y en la Universidad de la Coruña, donde es profesor del departamento de Psicología desde el año 2006. Cuenta con más de 30 publicaciones en libros y revistas científicas y con numerosas contribuciones a congresos científicos. Sus líneas de investigación se desarrollan en el campo de la psicología social con especial énfasis en el estudio de los valores motivacionales desde una perspectiva cultural.

\section{Mercedes Fernández Antón}

Doctora por la Universidad de A Coruña, licenciada en Ciencias Políticas y Sociología y en Filosofía y Ciencias de la Educación por la Universidad Complutense. Profesora Titular en la Universidad Complutense. Estancias como investigadora o profesora invitada en: Universidad de Auckland, Instituto de Investigaciones Sociales de la UNAM, Universidad de El Cairo, entre otras. Participación en más de una veintena de proyectos de investigación evaluados y financiados en convocatorias públicas, y como autora o coautora ha realizado un amplio número de publicaciones. Miembro fundador de la primera asociación de sociólogos de España y de la Federación Española de Sociología. Pertenece a la red de investigadores REYCIT, con sede en la UNAM, así como a otros grupos y comités de investigación. 